Www.jmscr.igmpublication.org

Index Copernicus Value: 79.54

ISSN (e)-2347-176x ISSN (p) 2455-0450

crossrefDOI: https://dx.doi.org/10.18535/jmscr/v7i2.85

Journal Of Medical Science And Clinical Research

IGM Publication

An official Publication of IGM Publication

\title{
Ocular manifestations in adult leukemia patients in a tertiary centre: A cross sectional study
}

\author{
Authors \\ Dr Swathi Karuppali ${ }^{1}$, Dr Arun Kumar Ayoor ${ }^{2 *}$, Dr Abdul Majeed K. ${ }^{3}$, \\ Dr Padma B Prabhu ${ }^{4}$ \\ ${ }^{1}$ Senior Resident, Dept of Ophthalmology, Government Medical College, Kozhikode \\ ${ }^{2}$ Professor, Dept of Ophthalmology, Govt Medical College, Kozhikode \\ ${ }^{3}$ Professor and Chief of Hematolgy Department, Dept of Medicine, Govt Medical College, Kozhikode \\ ${ }^{4}$ Associate Professor, Dept of Ophthalmology, Govt Medical College. Kozhikode \\ *Corresponding Author \\ Dr Arun Kumar Ayoor \\ Professor, Dept of Ophthalmology, Govt Medical College, Kozhikode, India
}

\begin{abstract}
Background: Leukemias are clonal neoplastic disorders of the hematopoetic stem cells in which immature cells infiltrate the blood stream and various tissues of the body. Eye is one such structure which is commonly involved either primarily by direct infiltration by leukemic cells or secondary to changes in the haematological parameters. Hence detailed ocular examination should be an essential part of treatment of leukemias to identify early changes.

Objectives: To study the various ocular manifestations in adult leukemia patients.

Methods: A cross sectional descriptive study was conducted among 200 eyes of 100 diagnosed leukemia patients who presented to hematology and ophthalmology department of Government Medical College Kozhikode. The patients were divided into various types of leukemia and detailed ocular examination was conducted. The anterior and posterior segment manifestations in each type of leukemias were noted .The observed findings were analysed using SPSS version 16.

Results and Discussion: Out of the 100 patients, there were 47 males and 53 females. The mean age of the study group was $38.35 \pm 19.83$ years. Acute myeloid leukemia was the commonest type (51\%). 61\%of the patients were symptomatic with defective vision being the commonest followed by watering as their chief complaints. Anterior segment manifestations were present in $76 \%$ patients with conjunctival congestion followed by pallor being the commonest. Posterior segment involvement was noted in 34\% patients of which majority had retinal haemorrhages followed by papilledema and optic disc cupping. These findings were more in the myeloid group who again had poorer visual acuity. Some patients developed drug related complications for which further detailed evaluation is needed to confirm the same in view of the multidrug regimens on which these patients are on.

Conclusion: High prevalence of ocular manifestations in the leukemic patients can be a cause of ocular morbidity and vision loss in these subjects. These findings may also be an early indicator of relapse. Hence regular ophthalmic assessment and early diagnosis can improve the disease outcome.

Keywords: Leukemia; ocular manifestations; retinal hemorrhage.
\end{abstract}




\section{Introduction}

Leukemias are clonal proliferative disorders of hematopoetic stem cells characterised by diffuse infiltration by the immature neoplastic cells in the peripheral blood, tissues and organs. Broadly classified in to four main categories based on the cell type being predominantly involved lymphopoetic or myelopoietic and based on clinical course as acute and chronic.

Eye involvement in leukemias can be either direct infiltration by the neoplastic cells or by secondary manifestations of the disease per se. Also these patients are more vulnerable to infections either due to their immunosuppressive state induced by the antineoplastic drugs or by the impaired hematopoesis. Understanding of ocular changes in leukemia is important because the eye is the only site where the leukemic involvement of nerves and blood vessels can be observed directly. Liebreich first described leukemic retinopathy in the 1860 s.

The published data regarding ocular manifestations in leukemias from various geographical areas reveal the importance of early detection of ocular and orbital lesions as they are reported to be the third most frequent extramedullary locations of acute leukemias after meninges and testis. Improved survival rates has led to the variability in the ocular presentations in the form of side effects of treatment. The present study was undertaken to describe the ocular manifestations in adult leukemia patients as Leukemic ophthalmopathy and co-morbid ocular disease contribute significantly to vision loss. These findings have clinical implications in the management of leukemia.

\section{Aim of the Study}

To study the various ocular manifestations in adult leukemia patients.

\section{Subjects and Methods}

All diagnosed adult leukemia patients presenting to the hematology department at a tertiary hospital for a period of 18 months from December
2014 and were willing to participate in the study, were prospectively analyzed and included in this study. The diagnosis of leukemia was confirmed based on bone marrow aspiration and peripheral smear. Further subdivision of leukemia into myelogenous and lymphocytic was made using cytomorphology and a combination of cytochemistry and immunophenotyping methods. Children below 12 years of age and patients with other diagnosed lymphoproliferative disorders like myelodysplastic syndromes, lymphomas, and hemoglobinopathies were excluded from this study. Patients with any pre existing primary retinal pathologies were also excluded.This study adheres with the tenants of the declaration of Helsinki.

Data on subjects demography, clinical history, chemotherapy received, hemoglobin, platelet, and white blood cell count were collected. visual acuity was recorded where possible using Snellen charts. Anterior segment examination was done using a flashlight and slit-lamp Fundus examination was done by slit lamp biomicroscopy, binocular indirect ophthalmoscopy, after dilating pupils with $1 \%$ tropicamide eye drops.

The collected data were entered to and statistical analysis was done using the SPSS version 16 (SPSS Inc., Chicago, Illinois, USA). An initial frequency count and percentages were obtained for all the data. All $P<0.05$ were accepted as statistically significant.

\section{Results}

The study group included 200 eyes of 100 diagnosed leukemia patients receiving treatment from the institute. Male female ratio was 4.7:5.3. The mean age of the study group was 38.35 years (SD 19.83). The mean haemoglobin value was $9.45 \mathrm{gm} / \mathrm{dl}$, white blood cell count was $8140.5 \mathrm{cells} / \mathrm{mm}^{3}$ and platelet count 2.65 lakh.

Patients were grouped into acute and chronic leukemia with myeloid and lymphoid subtypes. The distribution of cases based on the type of leukemia is given in figure 1. Majority were cases of AML. 


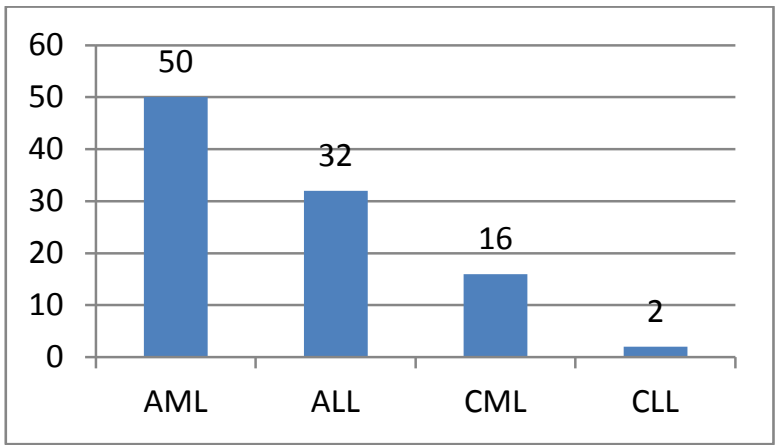

Figure 1 Distribution of Patients Based on Type of Leukemia

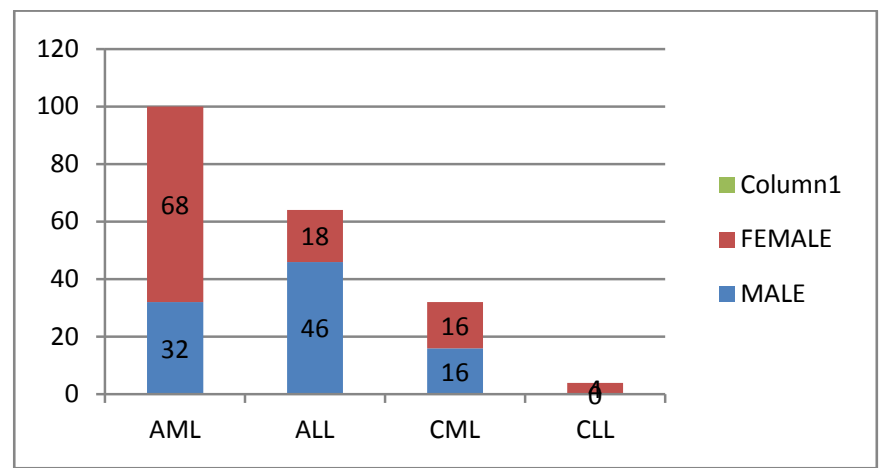

Figure 2 Gender Based Distribution of Cases
The gender distribution among various types of leukemia is given in figure 2. This showed a female preponderance for AML patients ratio of $1: 2.1$. The male female ratio among ALL was 2.5:1. CML cases showed equal gender distribution. All cases with CLL were females. These findings were statistically significant ( $p$ 0.001 )

Ocular symptoms were observed among $61 \%$ of cases. These included watering $(n=20)$, defective vision $n=22)$, redness $(n=7)$ and diplopia $(n=5)$. 25 eyes had multiple symptoms. The distribution of the types of leukemia based on their symptoms is given in table 1. Defective vision was the chief complaint among majority of AML,CML and CLL patients. Ocular surface symptoms were common among ALL cases.

These findings were statistically significant ( $\mathrm{p}$ $0.038)$

Table 1 Symptom Wise Distribution of Cases

\begin{tabular}{|l|c|c|c|c|}
\hline SYMPTOMS & AML & ALL & CML & CLL \\
\hline NO & 60 & 44 & 15 & 2 \\
\hline WATERING & $10(10 \%)$ & $6(9.35)$ & $4(12.5 \%)$ & 0 \\
\hline DEFECTIVE VISION & $12(12 \%)$ & 0 & $8(25 \%)$ & $2(50 \%)$ \\
\hline REDNESS & $3(3 \%)$ & $2(3.1 \%$ & $2(6.25 \%)$ & 0 \\
\hline DIPLOPIA & $3(3 \%)$ & $2(3.1)$ & 0 & 0 \\
\hline WATERING+REDNESS & $3(3 \%)$ & $4(6.2 \%)$ & $2(6.25 \%)$ & 0 \\
\hline DEFECTIVEVISION+REDNESS & $3(3 \%)$ & $6(9.3 \%)$ & 1 & 0 \\
\hline DEFECTIVEVISION+DIPLOPIA & $6(6 \%)$ & 0 & $0(3.12 \%)$ & 0 \\
\hline
\end{tabular}

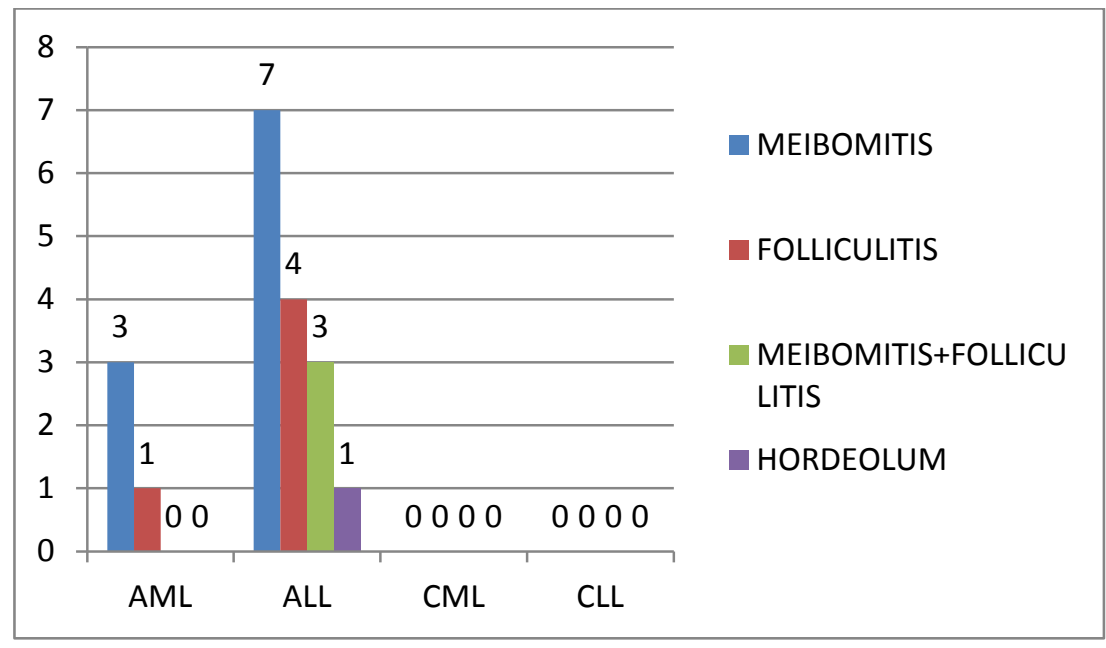

Figure 3 Distributions of Lid Signs

19 eyes showed lid signs. The distribution of cases based on the lid signs is given in figure 3.lid signs were present only among acute leukemias $(9.5 \%)$.
Meibomitis was the most prevalent finding $(\mathrm{n}=10)$. These observations were statistically significant $(\mathrm{p}=0.027)$. 


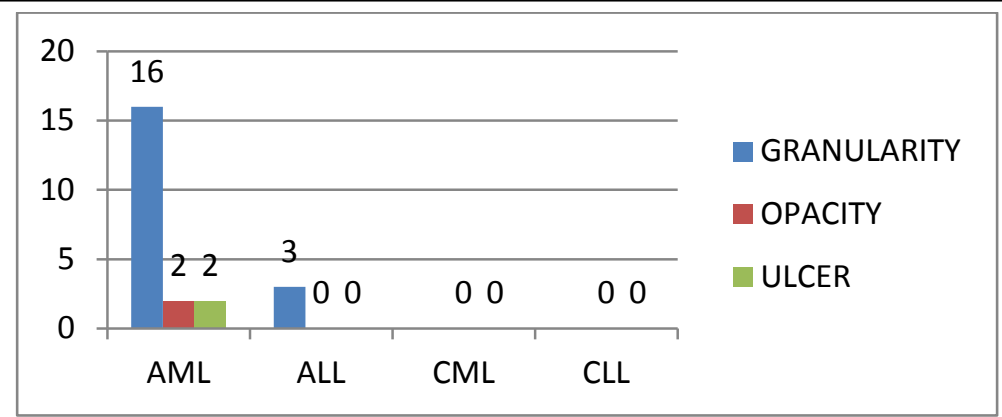

Figure 4 Distributions of Corneal Signs

23 eyes showed corneal signs.the distribution of cases based on corneal findings are given in figure 4.corneal epithelial granularity was the most common finding $(\mathrm{n}=19)$.

Table 2 Distributions of Conjunctival Findings

\begin{tabular}{|l|c|c|c|c|}
\hline & AML & ALL & CML & CLL \\
\hline congestion & 17 & $9(14 \%)$ & $8(25 \%)$ & 0 \\
\hline Subconjunctival hemorrhage & 5 & $5(7.8 \%)$ & $10(31.2 \%)$ & 0 \\
\hline pallor & 18 & $6(9.3 \%)$ & $3(9.3 \%)$ & 0 \\
\hline $\begin{array}{l}\text { Congestion and subconjunctival } \\
\text { hemorrhage }\end{array}$ & 2 & 0 & 0 & 0 \\
\hline $\begin{array}{l}\text { Subconjunctival hemorrhage and } \\
\text { pallor }\end{array}$ & 7 & $3(4.6 \%)$ & 0 & 0 \\
\hline
\end{tabular}

The distribution of cases with conjunctival signs are given in table 2. 81 eyes showed findings and these were conjunctival congestion $(n=34)$ subconjunctival hemorrhage $(\mathrm{n}=20)$, pallor $(\mathrm{n}=27) .12$ patients had multiple signs. Pallor was the commonest finding in AML patients followed by conjunctival congestion while subconjunctival
These findings were seen only in acute leukemias. $(\mathrm{p}=0.08)$

Table 3 Distribution of Retinal Findings

\begin{tabular}{|l|c|c|c|c|}
\hline RETINAL FINDING & AML & ALL & CML & CLL \\
\hline normal & 63 & 48 & 21 & 0 \\
\hline Superficial retinal hemorrhage & 8 & 6 & 2 & 0 \\
\hline Vitreous hemorrhage & 6 & 0 & 3 & 0 \\
\hline Optic disc cupping & 8 & 6 & 0 & 4 \\
\hline papilledema & 12 & 2 & 0 & 0 \\
\hline white centered haemorrhage & 2 & 2 & 2 & 0 \\
\hline Superficial and white centered haemorrhages. & 1 & 0 & 1 & 0 \\
\hline Pigment Epithelial Defect & 0 & 0 & 3 & 0 \\
\hline
\end{tabular}

Retinal signs were present in 68 eyes and these were superficial retinal hemorrhage $(n=16)$, vitreous hemorrhage $(n=9)$ optic disc cupping $(\mathrm{n}=18)$ papilledema $(\mathrm{n}=14)$ white centered hemorrhage $(n=6)$ retinal pigment epithelial defect. $(n=3)$. The distribution of cases based on retinal findings is given in table .3.optic nerve head changes $(16 \%)$ were present mainly in AML hemorrhage was the commonest in CML patients. These findings were statistically significant $(\mathrm{p}=0.005)$

Pupillary abnormality was present in 17 eyes and majority belonged to AML group $(n=14)$.this finding was statistically significant $(\mathrm{p}=0.03)$ and CLL patients whereas hemorrhagic manifestations in ALL and CML patients(16.5\%). Retinal pigment epithelial defect in 9.3\%of CML patients. all patients with CLL showed optic disc cupping and none of the patients in chronic leukemia group developed papilledema. these findings were statistically significant $(\mathrm{p}=0.001)$ 
Table 4 Distribution of Visual Acuity

\begin{tabular}{|c|c|c|c|c|c|c|}
\hline & \multicolumn{4}{|c|}{ TYPE } & \multirow[b]{2}{*}{ Total } \\
\hline & & AML & ALL & CML & CLL & \\
\hline \multirow[t]{11}{*}{ vision } & $6 / 6$ & 52 & 55 & 10 & 0 & 117 \\
\hline & $6 / 9$ & 10 & 4 & 6 & 0 & 20 \\
\hline & $6 / 12$ & 12 & 3 & 6 & 0 & 21 \\
\hline & $6 / 18$ & 9 & 1 & 6 & 2 & 18 \\
\hline & $6 / 24$ & 1 & 1 & 1 & 0 & 3 \\
\hline & $6 / 36$ & 3 & 0 & 1 & 2 & 6 \\
\hline & $6 / 60$ & 4 & 0 & 0 & 0 & 4 \\
\hline & $5 / 60-3 / 60$ & 1 & 0 & 0 & 0 & 1 \\
\hline & $2 / 60-1 / 60$ & 5 & 0 & 1 & 0 & 6 \\
\hline & $<1 / 60$ & 3 & 0 & 1 & 0 & 4 \\
\hline & Total & 100 & 64 & 32 & 4 & 200 \\
\hline
\end{tabular}

The distribution of visual acuity among the 4 types of leukemia is given in table 4.significant visual loss $<6 / 18$ was observed in 24 eyes majority of them belonging to AML group $(n=17)$.gross visual loss, 3/60 was seen in 8 eyes of AML and 2 eyes of CML patients. lymphoid type of leukemia presented with relatively better visual acuity than myeloid series. These findings were statistically significant. $(\mathrm{p}=0.000)$
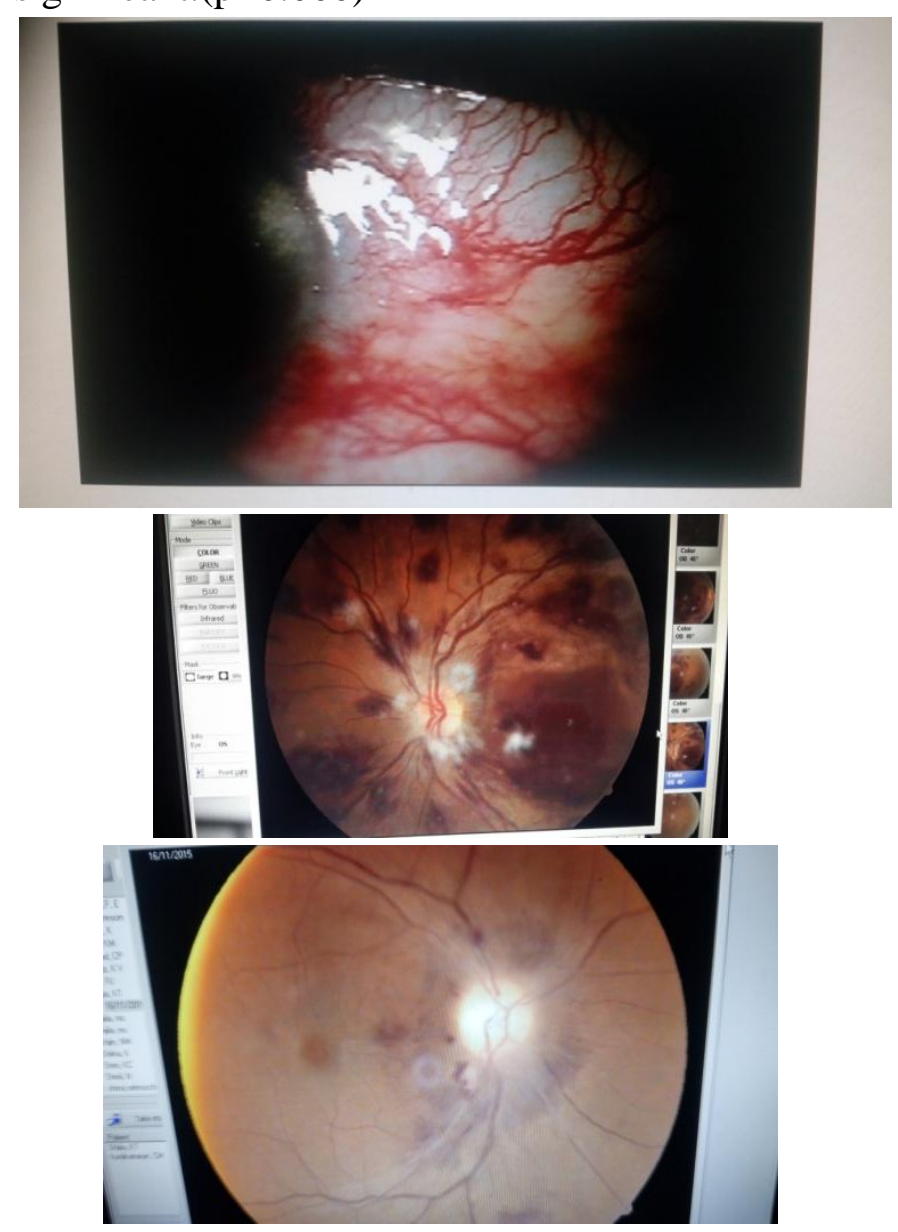

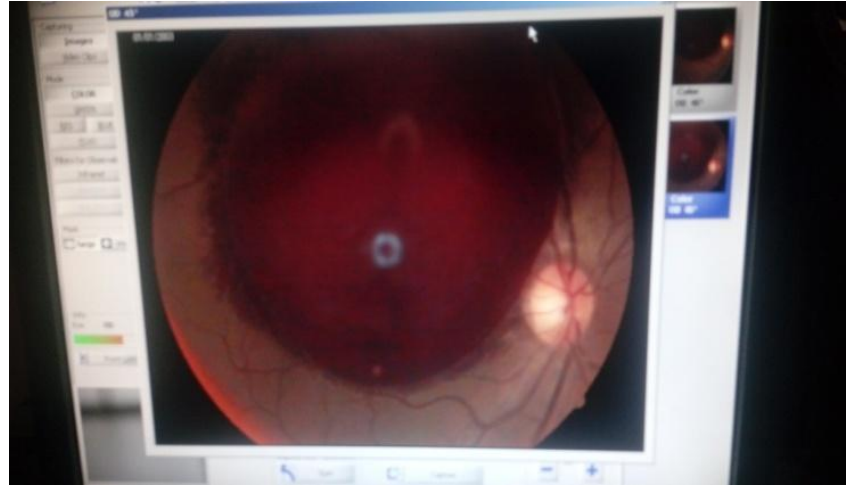

\section{Discussion}

Leukemias are now emerging as a leading cause of mortality and morbidity in the world and the knowledge of its multitude of ocular involvement is necessary for the early recognition and treatment as well as for detection of post treatment relapse. With the advent of newer chemotherapeutic modalities, the ocular manifestations are also showing a changing trend either in the form of toxicity to drugs or immunosuppression. This study cohort showed a preponderance of females $53 \%$. This was in contrast to to reports from Ethiopia ${ }^{(1)}$ Malaysia $^{(2)}$. the previous studies has explained their observed sex distribution due to prevalence pattern of leukemia or the established pro-male gender inequity in access to healthcare. This seems to suggest the the vanishing gender inequality in acquiring treatment in the current study area.The age of the patient ranged from 13 to 80 years with a mean age of 38.35 and standard deviation of 19.839. Comparable ranges were stated by koshy 
et al (mean $=39.73 \pm 22.1)^{(5)}$ and Reddy and Jackson (mean age 34.6 years) ${ }^{(4)}$ in their studies done on acute leukemia. Leukemia may present with or be associated with ocular disorders. Various studies has reported prevalence of ocular involvement to be between $9 \%$ and $90 .^{(1)(2)}$ This wide range of involvement might be attributed to the disease course and presence of transient ocular findings related to the treatment. Clinical and histopathologically the various types of leukemia may also present with different spectra of ocular findings.

The patients were recruited into the study when they had already been diagnosed with leukemia irrespective of whether they had ocular symptoms or not. The study sample was divided according to the type of leukemia based on their bone marrow report and classified into the 4 major groups of leukemias viz acute myeloid, acute lymphoid and chronic myeloid and chronic lymphocytic .100 patients were screened for the presence of ocular involvement.

Among the 100 patients 51 were diagnosed with acute myeloid leukemia, 31 as acute lymphoid leukemia, 16 as chronic myeloid and 2 with chronic lymphocytic leukemia. Similar observations were noted by Koshy et al in a study conducted at Ludhiana ${ }^{(5)}$.

Eighty six percent of the patients were already receiving chemotherapy. $61 \%$ of the patients had symptoms. Among the symptoms commonest was defective vision(11\%) followed bywatering $(10 \%)$ .Next common being redness (3.5\%) and diplopia for $2.5 \%$. Defective vision was the chief complaint among majority of AML,CML and CLL patients. Ocular surface symptoms were common among ALL cases. These findings were statistically significant ( $\mathrm{p}$ 0.038).in a similar study conducted by Renu et al at the department of ophthalmology Dehradun, ocular manifestations were noted in $40(39.3 \%)$ out of the 102 patients $^{(6)}$ The manifestations can be broadly divided as anterior segment and posterior segment manifestations. The anterior segment involvement is described here under headings of lid involvement, cornea and conjunctival involvement and posterior segment under retinal involvement.

Among the 100 patients studied 76 percent had anterior segment involvement and 36\% had posterior segment involvement. However, in a similar study by Godswill et al and Ocheni et al among nigerian population, the spectrum of leukemic ophthalmopathy showed a preponderance of posterior $86 \%$ over anterior segment manifestations. These resulted predominantly from secondary hematologic complications caused either by the systemic leukemia or its treatment rather than primary leukemic ocular infiltration ${ }^{(7)}$. Conjunctival findings were present in $40.5 \%$ of the sample . Pallor was the commonest finding in AML patients followed by conjunctival congestion while subconjunctival hemorrhage was the commonest in CML patients. These findings were statistically significant $\quad(p=0.005)$.Similar conjunctival findings were reportedby Godswill et al (34\%.)

Corneal findings were present in $11.5 \%$ which was exclusively in acute leukemias and this observation was statistically significant. A notable observation was in the acute myeloid group on cytarabine therapy who developed fine epithelial granularity while on chemotherapy. Case reports by lochhead et al and hopen et al has reported the occurrence of corneal cytotoxicity with both high and low dose regimens of cytosine arabinoside and the the need for prophylactic corticosteroid eye drops while on cytosine therapy to prevent formation of epithelial microcysts ${ }^{(8)(9)}$

Lid findings were more in the ALL subgroup (8\%). Posterior segment findings were present in $34 \%$ of the study group .Commonest findings were retinal haemorrhages in 20\%. Other significant findings were papilledema in $7 \%$, cupping of optic disc in $9 \%$ and retinal pigment epithelial defect in $2 \%$.While retinal haemorrhages were present irrespective of the subtype of leukemias, the presence of papilledema was mainly to the AML subgroup6\%. 
The posterior segment manifestations were predominantly due to secondary hematological complications caused either by the systemic leukemia or its treatment rather than primary leukemic ocular infiltration ${ }^{(3)(13))}$

Cupping of the optic disc was another significant finding which can be attributed to the impact of long term systemic corticosteroid use in conjunction with the chemotherapeutic regimens. In a study conducted by yamshita et al in 2010 among 5 children with acute lymphoblastic leukemia on corticosteroids, it was noticed that all developed significant glaucomatous damage of optic nerve head with raised intraocular pressure. ${ }^{(10)}$ However no similar study could be obtained pertaining to disc changes in adult population with leukemia and hence further evaluation for the confirmation of glaucoma in these subjects are needed to confirm the same.

There were only 2 patients with CLL and both showed optic disc cupping. Study by Buchan et al in 2003 among 25 CLL patients followed over a period of 6 months demonstrated that ocular involvement in CLL is uncommon, which is a reflection of the indolent course of CLL as compared to other leukemias ${ }^{(11)}$. The presence of cupping in these two patients needs further evaluation to rule out any glaucomatous damage or any secondary insult to the optic nerve due to drugs or disease per se. The limited number of patients in the CLL group during study also limits the interpretation of the disc change since there were no available data from the previous studies to support the same.

Regarding the visual acuity, it was observed that patients with lymphoid leukemias had better acuity and this could be attributed to more number of patients in the myeloid series who had vitreous hemorrhage and disc changes. optic nerve involvemant indirectly reflected from the sluggish pupillary response was also more in the myeloid group.

\section{Conclusion}

It can be observed that acute myeloid leukemias present with more severe ocular manifestations Leukemic patients may harbour varied manifestations in the eye majority of which may remain unnoticed. These patients can present to the ophthalmologist with visual symptoms either due to the disease or as a side effect of treatment. Definite clinical signs related to leukemic infiltration can be present on examination even in the absence of visual symptoms.

Moreover a prompt recognition of the ocular manifestations is crucial because of the poorer prognosis associated with ocular involvement and to identify possible extramedullary disease. Therefore all leukemia patients should have an ophthalmic assessment at diagnosis and periodically

\section{Reference}

1. Alemayehu W, Shamebo M, Bedri A, Mengistu Z. Ocular manifestations of leukaemia in Ethiopians. Ethiop Med J 1996;34:217-24.

2. Reddy SC, Jackson N, Menon BS. Ocular involvement in leukemia - a study of 288 cases. Ophthalmologica 2003;217:441-5.

3. Schachat AP, Markowitz JA, Guyer DR, Burke PJ, Karp JE, Graham ML. Ophthalmic manifestations of leukemia. Arch Ophthalmol 1989;107:697-700.

4. Reddy SC, Jackson N. Retinopathy in acute leukaemia at initial diagnosis: correlation of fundus lesions and haematological parameters. Acta ophthalmologica Scandinavica. 2004 Feb 1;82(1):81-5.

5. Koshy J, John MJ, Thomas S, Kaur G, Batra N, Xavier WJ. Ophthalmic manifestations of acute and chronic leukemias presenting to a tertiary care center in India. Indian journal of ophthalmology. 2015 Aug;63(8):659.

6. Dhasmana R, Prakash A, Gupta N, Verma $\mathrm{S}$ K. Ocular manifestations in leukemia 
and myeloproliferative disorders and their association with hematological parameters. Ann Afr Med 2016;15:97-103

7. Eze BI, Ibegbulam GO, Ocheni S. Ophthalmic manifestations of leukemia in a tertiary hospital population of adult Nigerian Africans. Middle East African journal of ophthalmology. 2010 Oct;17(4):325

8. Lochhead J, Salmon JF, Bron AJ. Cytarabine-induced corneal toxicity. Eye. 2003 Jul 1;17(5):677-8

9. Hopen G, Mondino BJ, Johnson BL, Chervenick PA. Corneal toxicity with systemic cytarabine. American journal of ophthalmology. 1981 Apr 30;91(4):500- 4.

10. Yamashita T, Kodama Y, Tanaka M, Yamakiri K, Kawano Y, Sakamoto T. Steroid-induced glaucoma in children with acute lymphoblastic leukemia: a possible complication. Journal of glaucoma. 2010 Mar 1;19(3):188-90.

11. Buchan J, McKibbin M, Burton T. The prevalence of ocular disease in chronic lymphocytic leukaemia. Eye (Lond) 2003; 17:27-30

12. Kincaid MC, Green WR. Ocular and orbital involvement in leukemia. Surv Ophthalmol 1983;27:211-32. 